\title{
The Transformation of School Auditorium Sound Reinforcement System
}

\author{
Yang Zhang \\ Beijing Information Technology College, Beijing, 100070, China
}

\begin{abstract}
At present, most of the researches on sound reinforcement system problems are focused on the new building cases. This paper discusses a transformation problem with "architectural acoustics" and "the sound reinforcement". In order to solve the problems efficiently, we take a transformation of school auditorium as a case. The problems are presented as "Enhancing the volume easily causes the acoustic feedback even howling" and "Lack of language sound reinforcement clarity in reports and meetings." This paper proposes solutions for reference. Firstly, increase the sound absorption by the decoration of the wall and the ground surface. Secondly, improve the speaker layout to better the voice clarity. Each transformation of the acoustic field is the one and only, different problems should be analysed according to the actual situation so that we could achieve better the Auditory experience.
\end{abstract}

Keywords-auditorium; the sound reinforcement system; howling; acoustic feedback; clarity.

\section{INTRODUCTION}

The sound reinforcement system of auditoriums is a kind of widely used system which is suitable for drama, music performances and meetings. It is characterized by high index requirements, far transmission distance and large dynamic range. Auditorium acoustics design including the "architectural acoustics" and "the sound reinforcement". The former's function is to control the reverberation time and sound quality defects, the latter's role is to ensure that the acoustic field distribution in halls have enough sound level and be uniform. It should also be able to supply different acoustic effect to adapt to various demands. The two elements are indispensable to obtain a good art and sound effects.

At present, some auditoriums are the reconstruction of old buildings, due to space or funds, the architectural acoustics design were not considered adequately in the construction, so there were some problems in the configuration and adjustment of electro acoustic equipment, which resulting in the low clarity, acoustic image confusion, transmission gain low, distortion and other issues in auditorium sound reinforcement. That would result in greatly the quality impairment of sound reinforcement system of auditorium. Taking a school auditorium as a case, this paper analyzes the problems of the auditorium sound reinforcement system, and put forward the corresponding solutions.

\section{DISCUSSED PROBLEMS}

The school auditorium, a renovation from a canteen, is an area of about $800 \mathrm{~m}^{2}$, which now mainly used for reports and academic seminars, conferences, community art performances, large choral competitions, multimedia video projection and academy award ceremony and other activities. The architectural of the canteen, designed with no consideration for the auditorium sound reinforcement function, has smooth marble ground, both sides of large area lighting glass windows and ceiling without sound absorption treatment. Electro acoustic equipments available include 16-channel analogy mixer, noise gate, equalizer, power amplifier and speakers, with livelier microphones and dynamic microphones. The main reinforcement system was set according to the left and right channel, including four 15-inch two frequency full range loudspeaker systems, two 18-inch ultra low frequency loudspeakers and four 12-inch two frequency monitor wedge speaker.

There are two existing problems in actual use:

(1) Enhancing the volume easily causes the acoustic feedback even howling.

(2) Lack of language sound reinforcement clarity in reports and meetings.

For each analysis of the problem is as follows.

\section{A. The acoustic feedback even howling}

Acoustic feedback is the most troublesome thing in the the sound reinforcement process, the system which picking up the sound by microphone has a strong chance that feedback howling. It does great harm to microphone. Power amplifier will produce great power output because of self excitation, which may burn the power amplifier and other sound equipment. The sound pressure produced by acoustic feedback is so high that it will hurt the ears of the audience. The sound set out from the speakers gets into the microphones and is amplified again after once amplification, forming a signal superposition which will lead to acoustic feedback and howling, as shown in figure 1.

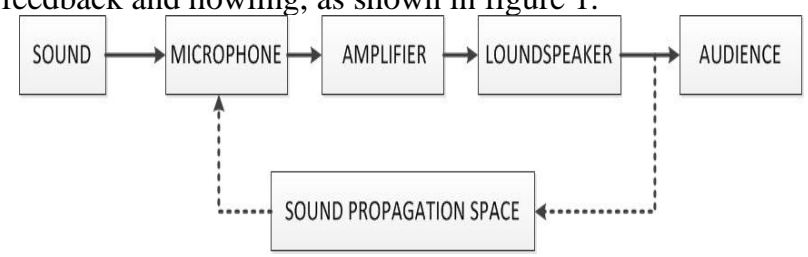

Figure 1. The process of acoustic feedback 
The howling emerges mostly because the reconstruction considered nothing about the basic requirements of architectural acoustic, with the smooth and hard surface of the walls and floors. Sound absorption decoration can reduce the the reflection of acoustic energy, with the electro acoustic equipment to solve the howling problem. Electro acoustic equipment commonly used is the feedback suppressor, its working principle is level suppression to the signal of certain frequency, the level of which exceeds the default value. This method is more efficient on the fixed microphone placement mode and conference amplifying than that in the performance on the stage. The actors are always in motion on the stage show, so that the frequency point tracking is very difficult. Besides, the signal of some frequency point level value overload because of not feedback but the larger dynamic range, the feedback suppressor would mistakenly the emergence of howling, and suppresses those signals resulting in the sound pressure collapse, disrupting the normal performance ${ }^{[1]}$.

\section{B. Lack of language sound reinforcement clarity in reports and meetings}

The site voice reinforcement should not only avoid the acoustic feedback, meeting the volume requirement, but also satisfy the voice articulation. Speech articulation depends mainly on the reverberation time, which closely related to the sense of subjective evaluation such as plump, warm, clear and space. The reverberation time of the hall for playing music should be longer so as to ensure the fullness of sound. The time of rooms for language Conference should be shorter in order to improve the speech intelligibility. The cinema also requires a short reverberation time because of needs of sound location. Sound reverberation time usually need to be designed in accordance with the following table.

TABLE I. REVERBERATION TIME

\begin{tabular}{|c|c|c|c|c|c|}
\hline \multirow{2}{*}{$\begin{array}{c}\text { Language } \\
\text { Conference }\end{array}$} & Cubage $/ \mathrm{m}^{3}$ & \multicolumn{2}{|c|}{$\leqslant 200$} & $200-500$ & $500-2000$ \\
\hline & $\begin{array}{c}\text { Reverberation } \\
\text { Time /S }\end{array}$ & \multicolumn{2}{|c|}{$0.3-0.5$} & $0.5-0.6$ & $0.6-0.8$ \\
\hline \multirow{3}{*}{ Cinema } & Cubage $/ \mathrm{m}^{3}$ & \multicolumn{2}{|c|}{$500-2000$} & $\geqslant 2000$ & \\
\hline & \multirow{2}{*}{$\begin{array}{c}\text { Reverberation } \\
\text { Time /S }\end{array}$} & Mono & $0.5-0.8$ & $0.8-1.2$ & \\
\hline & & Stereo & $0.4-0.6$ & $0.6-1.0$ & \\
\hline \multirow[b]{2}{*}{$\begin{array}{c}\text { Dancing } \\
\text { hall }\end{array}$} & Cubage $/ \mathrm{m}^{3}$ & \multicolumn{2}{|c|}{500} & 1000 & 2000 \\
\hline & $\begin{array}{c}\text { Reverberation } \\
\text { Time /S } \\
\end{array}$ & \multicolumn{2}{|c|}{0.9} & 1.0 & 1.1 \\
\hline
\end{tabular}

The area of the auditorium in the case is about $800 \mathrm{~m}^{2}$, its cubage is about $2000 \mathrm{~m}^{3}$. And the function involves language reinforcement, theatrical performances and others. According to table 1 , the reverberation time should be reasonably about 1 second. But now it is at least 2.5 seconds or more, which not only decline the clarity of sound reinforcement, also has a seriously negative effect on performance.

\section{SUGGESTIONS ABOUT THE TRANSFORMATION}

The transformation should solve the two problems as howling and clarity on the premise of saved cost, ensuring the system stability and high cost performance.

\section{A. Architectural acoustics transformation}

The decoration of sound absorption could change the reverberation time effectively of the auditorium. The ceiling and the wall here are suggested stronger sound-absorbing, side walls do not need the transformation to strengthen the reflection to achieve more music fullness [2]. The ceiling strong absorption could absorb the previous 3 reflection, which is similar to the whole absorption. The absorption of back wall would also reduce the reflection greatly. The voice would be as same clear in a room without ceiling and walls as outdoors. Besides add carpets and curtains, Specific recommendations are presented as follows.

- Do sound absorption decoration to the ceilings on audience and stage. Do the decoration to the back wall in order to increase the back cavity, which could absorb not only high frequency but also the resonance sound of low frequency, as shown in figure 2.
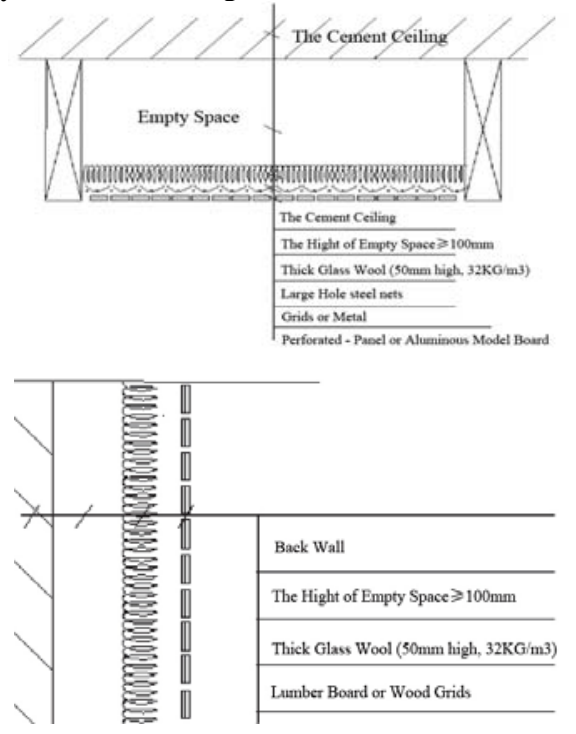

Figure 2. Decoration to the ceilings and the back wall

- Add reflection dispersions to side wall, as shown in figure 3.

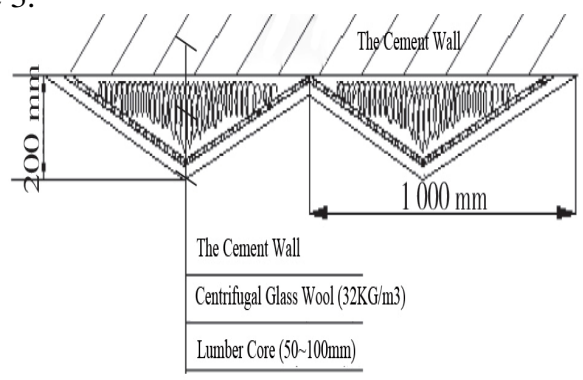

Figure 3. Decoration to the side wall

\section{B. The loudspeakers and the layout}

Selection and layout of the speakers largely determine the success of a field. It should ensure both that the sound pressure distribution of all the audience is uniform, and that the sound source direction of the majority audience is right, 
which means that the source direction of audience hearing is consistent with the lecturer. The original loudspeaker configuration and setup neither make the sound reinforcement system work well nor meet the demand of field uniformity.

There are original four 15 inch two divider full frequency speakers with the frequency response of 68 $\mathrm{Hz}-19 \mathrm{kHz}( \pm 3 \mathrm{~dB})$, the maximum sound pressure level of $131 \mathrm{~dB}$ and the coverage angle of $60 \mathrm{o} \times 40 \mathrm{o}$. Two of them are advised to be a central array as main amplification, the other two be placed as left and right speaker in the side to meet the reinforcement need of audience area.

Column loudspeaker is suggested to be used as the main speaker for its brilliant intelligibility. It is a line array of a certain number of direct type loudspeakers that be mounted in a column shaped box. Column loudspeaker characteristics far radiation and high efficiency, which could improve sound energy density of far field. The directivity of column loudspeaker is in the vertical plane and narrows for the interference effect caused by the phase difference of longitudinal speaker to a point, forming a sharp beam. The directivity in the horizontal plane of column loudspeaker is the same round as a regular speaker, which is between the point source and line one.

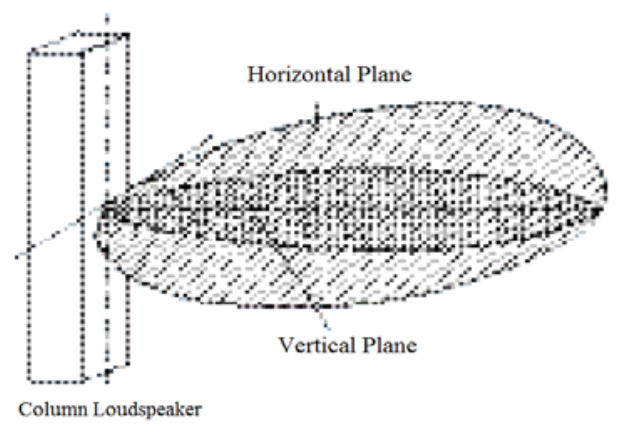

Figure. 4. The directivity of column loudspeaker
The installation position of column loudspeaker indoor should be determined according to the length and height of the hall. Angle installation is generally used. The inclination angle with the wall is suggested between $8^{\circ}$ and $20^{\circ}$, the central axis projection point should between $2 / 3$ and $3 / 4$ of the full-length in the audience, as shown in figure 5.

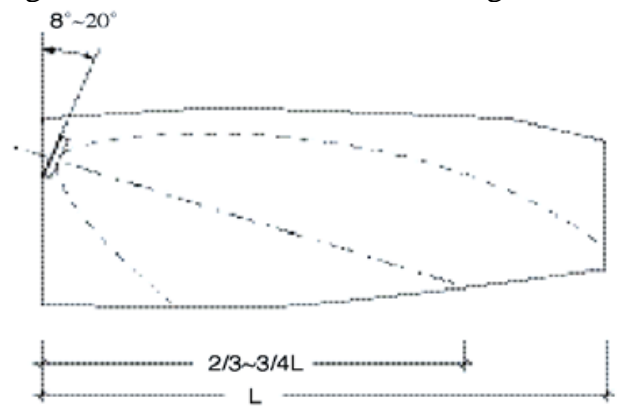

Figure. 5. The installation position of column loudspeaker ${ }^{[3]}$

\section{CONCLUSIONS}

With the development of education, more and more buildings such as the training room, multifunctional hall, lecture hall and auditorium need to be reconstructed to meet the expanding need of teaching and training. The amplifying function and various aspects of architectural acoustics conditions must be fully considered in the early reconstruction in order to get high quality sound.

\section{REFERENCES}

[1] Qiang Wang, The acoustic design of sound reinforcement system about the language clarity in the hall, AV Technology, 2, pp. 29-32, 2009.

[2] Jialong Yan, Focus On the construction of auditorium sound reinforcement system for teaching, $A V$ Technology, 6, pp. 27-29, 2012

[3] Yizhen Wang, Analysis on controllable directional column loudspeaker, Audio Engineering, 34(6), 2010. 